\title{
Symplectic Maps and Chromatic Optics in Particle Accelerators
}

\author{
Yunhai Cai \\ SLAC National Accelerator Laboratory \\ Menlo Park, CA 94025
}

\begin{abstract}
We have applied the nonlinear map method to comprehensively characterize the chromatic optics in particle accelerators. Our approach is built on the foundation of symplectic transfer maps of magnetic elements. The chromatic lattice parameters can be transported from one element to another by the maps. We introduce a Jacobian operator that provides an intrinsic linkage between the maps and the matrix with parameter dependence. The link allows us to directly apply the formulation of the linear optics to compute the chromatic lattice parameters. As an illustration, we analyze an alternating-gradient cell with nonlinear sextupoles, octupoles, and decapoles and derive analytically their settings for the local chromatic compensation. As a result, the cell becomes nearly perfect up to the third-order of the momentum deviation.
\end{abstract}




\section{Introduction}

The linear optics in particle accelerators is well established by Courant and Snyder in the theory of alternating-gradient synchrotron [1]. It was extended to include the coupling between the horizontal and vertical planes by Edwards and Teng [2]. For the design of accelerators, Brown developed a first- and second-order matrix theory [3] that provided a powerful physics engine inside many codes, such as MAD [4] and ELEGANT [5]. The optics parameters introduced by Courant and Snyder can be easily computed and manipulated in these matrix codes. Therefore they are routinely used to design the linear optics in accelerators.

In chromatic optics, the key question is how the optical parameters depend on the momentum deviation $\delta$. Now, the computer codes can provide the numerical values for any given $\delta$. This capacity allows us to minimize the chromatic effects by numerically adjusting the settings of sextupoles. This kind of optimization is often achieved by running computer codes like HARMON [6], or SAD [7]. The numerical solutions found by computers are useful and often adequate. However they hardly provide any insights for the underlining physics.

The analytical approach is also developed using the perturbation theory. The first order chromaticity is treated in many textbooks, for example that of Edwards and Syphers [8]. The higher order effects are analyzed, for instance in a synchrotron light source with strong sextupoles [9]. A more general approach based on the differential algebra [10] and nonlinear normal form [11] can be found in the books of Berz [12] and Forest [13], respectively. In this paper, we will further extend these developments and apply the map method with a focus to the analysis and transport of the lattice parameters.

Our work is partially motivated by the need to develop the next generation storage rings. For high-energy physics, a circular Higgs factory [14] requires an extremely large momentum acceptance to accommodate the beamstrahlung radiation [15] generated by the colliding electron and positron beams near $120 \mathrm{Gev}$. As a synchrotron light source, an ultimate storage ring [16] reduces the natural emittance down to a few pico-meters and yet retains a large enough aperture for an efficient injection and good beam lifetime. With these motivations in mind, we will develop a method to analytically compute these parameters, trying to understand the chromatic effects better.

In the first part of the paper, we will mostly review the theory of linear 
optics in particle accelerators. In Sec. 2, we will introduce the concept of transfer maps with examples of a sector bending magnet and a thin-lens multipoles. Continuing to Sec. 3, we will outline the theory of linear optics and end with the linear normal form for the coasting beam.

In Sec. 4, the start of the second part, we will derive a transfer map relative to a dispersive orbit that defines the initial condition in a periodic beamline. With the periodic map, we will introduce a Jacobian operator to link the map with the matrix with dependence on $\delta$. Using the matrix, we will calculate the lattice parameters that characterize the chromatic optics in accelerators. Given the theory in Sec. 5, we analyze the chromatic optics for an alternating-gradient cell including sextupoles, octupoles, and decapoles. Finally, we will finish with some concluding remarks in Sec. 6 .

\section{Symplectic Maps}

It is well known $[17,18]$ that the dynamics of a charged-particle in accelerators can be described by tracking its canonical coordinates $z=\left(x, p_{x}, y, p_{y}, \delta, \ell\right)$, where $\delta=\left(p-p_{0}\right) / p_{0}$ is the relative momentum deviation and $\ell=v t, v$ is the velocity of the particle, and $t$ is the time of flight. For a magnetic element schematically illustrated in Fig. 1, its transfer map $\mathcal{M}$ can be obtained by solving the Hamilton's equations.

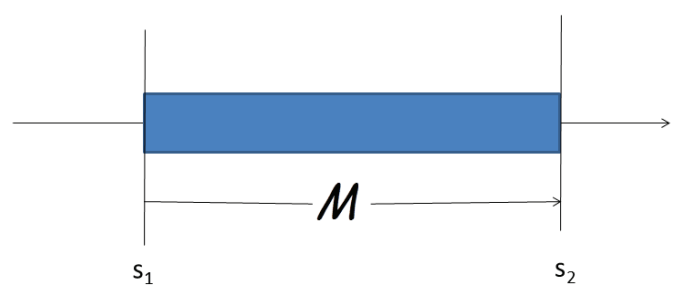

Figure 1: An element in beamline represented by its transfer map of the canonical coordinates $z=\left(x, p_{x}, y, p_{y}, \delta, \ell\right)$ from position $s_{1}$ to $s_{2}$.

The resulting map is symplectic, because its Jacobian,

$$
\mathcal{J}(\mathcal{M}) \equiv \frac{\partial\left(\mathcal{M}_{1}, \ldots, \mathcal{M}_{6}\right)}{\partial\left(z_{1}, \ldots, z_{6}\right)},
$$


satisfies the symplectic condition,

$$
\mathcal{J}(\mathcal{M}) \cdot J \cdot \mathcal{J}^{T}(\mathcal{M})=J
$$

where $\mathcal{J}^{T}$ denotes the transpose of $\mathcal{J}$ and the matrix $J$ is given by,

$$
J=\left(\begin{array}{rrrrrr}
0 & 1 & 0 & 0 & 0 & 0 \\
-1 & 0 & 0 & 0 & 0 & 0 \\
0 & 0 & 0 & 1 & 0 & 0 \\
0 & 0 & -1 & 0 & 0 & 0 \\
0 & 0 & 0 & 0 & 0 & 1 \\
0 & 0 & 0 & 0 & -1 & 0
\end{array}\right)
$$

There are many known transfer maps [19] for the magnets in accelerators. For example, the transfer map of a sector bend can be obtained by solving the Hamiltonian's equations with,

$$
H=\frac{p_{x}^{2}+p_{y}^{2}}{2(1+\delta)}-\frac{x \delta}{\rho},
$$

where $\rho$ is the bending radius. A general Hamiltonian of a sector bend with the path length $s$ as independent variable can be found in the paper by Ruth [17]. This simple Hamiltonian can be derived using the paraxial approximation. The map can be written as,

$$
\begin{aligned}
& \mathcal{M}_{1}=x+\frac{L}{1+\delta}\left(p_{x}+\frac{\theta \delta}{2}\right) \\
& \mathcal{M}_{2}=p_{x}+\theta \delta \\
& \mathcal{M}_{3}=y+\frac{L p_{y}}{1+\delta} \\
& \mathcal{M}_{4}=p_{y} \\
& \mathcal{M}_{5}=\delta \\
& \mathcal{M}_{6}=\ell+\theta x+\frac{L}{2(1+\delta)^{2}}\left[p_{x}^{2}+p_{y}^{2}+\theta(1+2 \delta)\left(p_{x}+\frac{\theta \delta}{3}\right)\right]
\end{aligned}
$$

where $L$ is the length and $\theta=L / \rho$ the bending angle of the dipole. Another type of the useful transfer maps is the kick, generated by a Hamiltonain that depends only on the transverse positions $x$ and $y$. For a combined thin 
quadrupole, sextupole, octupole, and decapole, the map is given by,

$$
\begin{aligned}
& \mathcal{M}_{1}=x, \\
& \mathcal{M}_{2}=p_{x}-\frac{x}{f}-\frac{\kappa}{2}\left(x^{2}-y^{2}\right)-\frac{o}{6} x\left(x^{2}-3 y^{2}\right)-\frac{\xi}{24}\left(x^{4}-6 x^{2} y^{2}+y^{4}\right), \\
& \mathcal{M}_{3}=y, \\
& \mathcal{M}_{4}=p_{y}+\frac{y}{f}+\kappa x y+\frac{o}{6} y\left(3 x^{2}-y^{2}\right)+\frac{\xi}{6} x y\left(x^{2}-y^{2}\right), \\
& \mathcal{M}_{5}=\delta, \\
& \mathcal{M}_{6}=\ell,
\end{aligned}
$$

where $f$ is the focusing (in the horizontal plane) length of quadupole and $\kappa, o, \xi$ are the integrated strengths of sextupole, octupole, and decapole respectively.

\section{$3 \quad$ Linear optics}

The linear optics of a beamline that consists of a sequence of elements is entirely determined by the R-matrix, which is the linear part of the map and can be computed as,

$$
\left.R \equiv \mathcal{J}(\mathcal{M})\right|_{z=0}
$$

Clearly from the definition, it is also symplectic. It is obvious that the $\mathrm{R}$ matrix of the beamline is given by a product of matrices,

$$
R=R_{n} \cdot \ldots R_{2} \cdot R_{1},
$$

where index 1 is for the first element seen by the charged particle in the beamline and $\mathrm{n}$ for the last. For a periodic system such as a storage ring, we can analyze the betatron motions by taking out the diagonal $2 \times 2$ matrix. For the horizontal motion, we have

$$
M=\left(\begin{array}{ll}
R_{11} & R_{12} \\
R_{21} & R_{22}
\end{array}\right),
$$

or for the vertical motion,

$$
M=\left(\begin{array}{ll}
R_{33} & R_{34} \\
R_{43} & R_{44}
\end{array}\right)
$$

It is easy to show that $M$ is a symplectic matrix as well and its determinant

equals one. This leads us to the Courant-Snyder parameters for the linear optics. 


\subsection{Courant-Snyder Parameters}

It is well known that the betatron motion at a location $s$ in a circular accelerator can be described by the Courant-Snyder parameters: $\beta(s), \alpha(s)$, and $\gamma(s)=\left(1+\alpha(s)^{2}\right) / \beta(s)[1]$

$$
M=\left(\begin{array}{ll}
\cos \mu+\alpha \sin \mu & \beta \sin \mu \\
-\gamma \sin \mu & \cos \mu-\alpha \sin \mu
\end{array}\right),
$$

where $\mu=2 \pi \nu$ is the phase advance and $\nu$ is the betatron tune. Given the matrix $M$, we can compute,

$$
\begin{aligned}
\beta & =M_{12} / \sin \mu, \\
\alpha & =\left(M_{11}-M_{22}\right) /(2 \sin \mu), \\
\gamma & =-M_{21} / \sin \mu,
\end{aligned}
$$

with $\mu=\cos ^{-1}\left(\left(M_{11}+M_{22}\right) / 2\right) . \quad \mu$ is uniquely defined within 0 and $2 \pi$ provided $\beta>0$. Moreover, the matrix $M$ can be factorized as

$$
M=U_{0} \cdot N \cdot U_{0}^{-1},
$$

where $N$ is a rotational matrix,

$$
N(\mu)=\left(\begin{array}{ll}
\cos \mu & \sin \mu \\
-\sin \mu & \cos \mu
\end{array}\right)
$$

and

$$
U_{0}=\left(\begin{array}{ll}
\sqrt{\beta} & 0 \\
\frac{-\alpha}{\sqrt{\beta}} & \frac{1}{\sqrt{\beta}}
\end{array}\right), U_{0}^{-1}=\left(\begin{array}{ll}
\frac{1}{\sqrt{\beta}} & 0 \\
\frac{\alpha}{\sqrt{\beta}} & \sqrt{\beta}
\end{array}\right) .
$$

$U_{0}^{-1}$ defines a transformation to the normalized coordinates and $U_{0}$ a transformation back. This transformation is not unique. In fact, a general solution is given by

$$
U=U_{0} \cdot N(\psi)
$$

where $\psi$ is a phase given by

$$
\psi=\tan ^{-1}\left(U_{12} / U_{11}\right),
$$

which can be interpreted as the phase advance of the betatron oscillation. We can show that the Courant-Snyder parameters are given by,

$$
\begin{aligned}
\beta & =U_{11}^{2}+U_{12}^{2}, \\
\alpha & =-\left(U_{11} U_{21}+U_{12} U_{22}\right) \\
\gamma & =U_{21}^{2}+U_{22}^{2},
\end{aligned}
$$


if the general solution is used.

Once we know the lattice functions $\beta, \alpha$, and $\gamma$ at a location $s_{1}, U_{0}\left(s_{1}\right)$ can be constructed using Eq. (3.9). We can then compute $U\left(s_{2}\right)$ by,

$$
U\left(s_{2}\right)=M_{(1 \rightarrow 2)} \cdot U_{0}\left(s_{1}\right),
$$

where $M_{(1 \rightarrow 2)}$ is the transfer matrix from $s_{1}$ to $s_{2}$. We can verify that $U\left(s_{2}\right)^{-1}$ is indeed the transformation to the normal coordinates at the location $s_{2}$ by,

$$
\begin{aligned}
& U\left(s_{2}\right)^{-1} \cdot M_{(2 \rightarrow 2)} \cdot U\left(s_{2}\right) \\
= & \left(M_{(1 \rightarrow 2)} \cdot U_{0}\left(s_{1}\right)\right)^{-1} \cdot M_{(2 \rightarrow 2)} \cdot\left(M_{(1 \rightarrow 2)} \cdot U_{0}\left(s_{1}\right)\right) \\
= & U_{0}\left(s_{1}\right)^{-1} \cdot M_{(1 \rightarrow 2)}^{-1} \cdot M_{(2 \rightarrow 2)} \cdot M_{(1 \rightarrow 2)} \cdot U_{0}\left(s_{1}\right) \\
= & U_{0}\left(s_{1}\right)^{-1} \cdot M_{(1 \rightarrow 1)} \cdot U_{0}\left(s_{1}\right) \\
= & N(\mu) .
\end{aligned}
$$

To complete the proof, we have to use the fact that $\mu$ is an invariant and does not depend on the location $s$. Of course, with $U\left(s_{2}\right)$ in hand, we can calculate the lattice functions $\beta\left(s_{2}\right), \alpha\left(s_{2}\right)$, and $\gamma\left(s_{2}\right)$ using Eq. (3.12). Then the phase in Eq. (3.11) gives the betatron phase advance. This procedure provides us a straightforward way to propagate the Courant-Snyder parameters $\alpha, \beta, \gamma$, and phase advance $\psi$ through a lattice. Clearly, the method is applicable to single-pass optics as well, if its initial condition is given.

We should point out that this method of computing the Courant-Snyder parameters was implemented in code TRACY [20]. Furthermore, if one carries out the procedure explicitly, the final results are identical to those derived long ago by Brown and Servranckx [21]. The reason we write it in great detail is that it can be easily extended to include the coupling, dispersion, and later as a function of $\delta$.

\subsection{Edwards and Teng coupling parameters}

In general, the transverse motion in a circular accelerator can be coupled between the horizontal and vertical planes, for example by skew quadrupoles

or solenoids. When there is coupling, we have to consider the $4 \times 4$ matrix,

$$
\mathbf{T}=\left(\begin{array}{cccc}
R_{11} & R_{12} & R_{13} & R_{14} \\
R_{21} & R_{22} & R_{23} & R_{24} \\
R_{31} & R_{32} & R_{33} & R_{34} \\
R_{41} & R_{42} & R_{43} & R_{44}
\end{array}\right)
$$


as a sub matrix of the R-matrix. It has been shown by Edwards and Teng [2] that $\mathbf{T}$ in a periodic and symplectic system can be decoupled by a similarity transformation

$$
\mathbf{T}=\mathbf{W} \cdot \mathbf{M} \cdot \mathbf{W}^{-1},
$$

where $\mathbf{T}, \mathbf{M}$, and $\mathbf{Z}$ are all $4 \times 4$ symplectic matrices. In particular, $\mathbf{M}$ is in a block diagonal form

$$
\mathbf{M}=\left(\begin{array}{cc}
M_{1} & 0 \\
0 & M_{2}
\end{array}\right)
$$

and $\mathbf{W}$ is in a "symplectic rotation" form

$$
\mathbf{W}=\left(\begin{array}{cc}
g I & \bar{W} \\
-W & g I
\end{array}\right)
$$

where $M_{1,2}, I, W$, and $\bar{W}$ are all $2 \times 2$ matrices. Here $I$ is the identity matrix, and $\bar{W}$ is defined as the symplectic conjugate of matrix $W$, namely $\bar{W}=-J_{2} \cdot W^{T} \cdot J_{2}$, where $J_{2}$ is the unit symplectic matrix

$$
J_{2}=\left(\begin{array}{cc}
0 & 1 \\
-1 & 0
\end{array}\right) .
$$

Moreover, the four-dimensional symplecticity requires that the sub-matrices $M_{1,2}$ are symplectic and $g^{2}=1-\operatorname{det} W$.

To find the matrices $M_{1,2}$ and $W$, we follow the approach by Edwards and Teng and first decompose the matrix $\mathbf{T}$ in terms of $2 \times 2$ matrices,

$$
\mathbf{T}=\left(\begin{array}{ll}
\mathbf{T}_{11} & \mathbf{T}_{12} \\
\mathbf{T}_{21} & \mathbf{T}_{22},
\end{array}\right)
$$

and then solve Eq. (3.16) for the coupling matrix,

$$
W=-\left(\overline{\mathbf{T}}_{12}+\mathbf{T}_{21}\right) /\left(g \operatorname{Tr}\left(M_{1}-M_{2}\right)\right),
$$

with $\operatorname{Tr}\left(M_{1}-M_{2}\right)= \pm \sqrt{\operatorname{Tr}^{2}\left(\mathbf{T}_{11}-\mathbf{T}_{22}\right)+4 \operatorname{det}\left(\overline{\mathbf{T}}_{12}+\mathbf{T}_{21}\right)}$ and,

$$
g=\sqrt{\frac{1}{2}\left(1+\frac{\left|\operatorname{Tr}\left(\mathbf{T}_{11}-\mathbf{T}_{22}\right)\right|}{\left|\operatorname{Tr}\left(M_{1}-M_{2}\right)\right|}\right)} .
$$

In the derivation, we have repeatedly used an identity, $W \cdot \bar{W}=\bar{W} \cdot W=$ $(\operatorname{det} W) I$. Having $W$, we easily find the other two matrices,

$$
\begin{aligned}
& M_{1}=\mathbf{T}_{11}-\bar{W} \cdot \mathbf{T}_{21} / g, \\
& M_{2}=\mathbf{T}_{22}+W \cdot \mathbf{T}_{12} / g .
\end{aligned}
$$


Since $M_{1,2}$ are symplectic, for each mode, we can use the Courant-Snyder parameters outlined in the previous section to represent them. Specifically, $\beta_{1,2}, \alpha_{1,2}, \gamma_{1,2}$ and $\mu_{1,2}$ can be computed using Eq. (3.12) for each mode respectively. For the coupled system, we need the additional four parameters $w_{1,2,3,4}$

$$
W=\left(\begin{array}{ll}
w_{1} & w_{2} \\
w_{3} & w_{4}
\end{array}\right)
$$

to describe the coupling matrix $W$. Hence we have ten independent parameters to specify the linearly coupled optics.

The transformation $\mathbf{A}_{0}^{-1}$ to the normal modes can be easily constructed by applying what we have learned in the previous section,

$$
\mathbf{T}=\mathbf{A}_{0} \cdot \mathbf{N} \cdot \mathbf{A}_{0}^{-1}
$$

where

$$
\mathbf{N}=\left(\begin{array}{ll}
N_{1} & 0 \\
0 & N_{2}
\end{array}\right)
$$

and

$$
\mathbf{A}_{0}=\left(\begin{array}{cc}
g I & \bar{W} \\
-W & g I
\end{array}\right) \cdot\left(\begin{array}{ll}
U_{01} & 0 \\
0 & U_{02}
\end{array}\right),
$$

$U_{01}$ and $U_{02}$ are the matrices constructed with Eq. (3.9) for mode 1 and 2 respectively. It is worth noting that the solution of Eq. (3.25) can be constructed [11] numerically using eigen values and eigen vectors of the matrix $\mathbf{T}$. These two methods yield identical numerical results in our study. However, this analytical approach allows us to later include the parameter dependence of $\delta$. Again, $\mathbf{A}_{0}$ is not unique, a general solution is given by,

$$
\mathbf{A}=\left(\begin{array}{cc}
g I & \bar{W} \\
-W & g I
\end{array}\right) \cdot\left(\begin{array}{ll}
U_{1} & 0 \\
0 & U_{2}
\end{array}\right)
$$

Now there are two phases that can be determined relative to the transformation in the other position. Here we should note that $\mathbf{A}$ is also a symplectic matrix.

Similar to the non-coupled case in the previous section, we can propagate the coupled lattice parameters by computing,

$$
\mathbf{A}\left(s_{2}\right)=\mathbf{T}_{(1 \rightarrow 2)} \cdot \mathbf{A}_{0}\left(s_{1}\right),
$$


where $\mathbf{T}_{(1 \rightarrow 2)}$ is the $4 \times 4$ transport matrix from position $s_{1}$ to $s_{2}$. Decomposing $\mathbf{A}\left(s_{2}\right)$ in terms of $2 \times 2$ matrices,

$$
\mathbf{A}=\left(\begin{array}{ll}
\mathbf{A}_{11} & \mathbf{A}_{12} \\
\mathbf{A}_{21} & \mathbf{A}_{22}
\end{array}\right)
$$

and knowing it in the form of Eq. (3.28), we find $g=\sqrt{\operatorname{det} \mathbf{A}_{11}}$, and

$$
U_{1}=\mathbf{A}_{11} / g, U_{2}=\mathbf{A}_{22} / g, W=-\mathbf{A}_{21} \cdot U_{1}^{-1} \text {. }
$$

Having $U_{i}$, we can calculate $\beta_{i}, \alpha_{i}, \gamma_{i}$ and the betatron phase $\psi_{i}$ using Eqs. (3.12) and (3.11).

We should point out that there are many papers on the topic of coupling. Among them, Sagan and Rubin wrote the most comprehensive paper [22], in which the derivation of the results in this section can be found.

\subsection{Dispersion}

So far, we have discussed the betatron motion of an on-momentum particle. The question we will address in this section is what if the particle has a slightly different momentum relative to the designed momentum and how can we describe its motion. Let's start with a general form of the R-matrix,

$$
R=\left(\begin{array}{llllll}
R_{11} & R_{12} & R_{13} & R_{14} & D_{1} & 0 \\
R_{21} & R_{22} & R_{23} & R_{24} & D_{2} & 0 \\
R_{31} & R_{32} & R_{33} & R_{34} & D_{3} & 0 \\
R_{41} & R_{42} & R_{43} & R_{44} & D_{4} & 0 \\
0 & 0 & 0 & 0 & 1 & 0 \\
E_{1} & E_{2} & E_{3} & E_{4} & R_{65} & 1
\end{array}\right)
$$

that describes a beamline containing only static magnets. Since the magnetic field cannot provide any energy to the charged particle, $\delta$ never changes in the static magnets. This leads to five zeros in the fifth row. The other zeros in the six column can be explained because of no dependency on the time

of flight. The rest of its form is entirely determined by the $6 \times 6$ symplectic condition in Eq. (2.2). Specifically, if we introduce a dispersive vector,

$$
\mathbf{D}=\left(\begin{array}{c}
D_{1} \\
D_{2} \\
D_{3} \\
D_{4}
\end{array}\right)
$$


and then the vector,

$$
\mathbf{E}=\left(\begin{array}{c}
E_{1} \\
E_{2} \\
E_{3} \\
E_{4}
\end{array}\right)
$$

it is given by,

$$
\mathbf{E}=\mathbf{T}^{T} \cdot \mathbf{J} \cdot \mathbf{D}
$$

where $\mathbf{T}$ is the matrix we have introduced in Eq. (3.15) of the previous section and itself has to be $4 \times 4$ symplectic. Here the matrix $\mathbf{J}$ is given by,

$$
\mathbf{J}=\left(\begin{array}{rrrr}
0 & 1 & 0 & 0 \\
-1 & 0 & 0 & 0 \\
0 & 0 & 0 & 1 \\
0 & 0 & -1 & 0
\end{array}\right)
$$

In addition to the ten parameters for the coupled betatron motion, we will have an extra five independent variables: $D_{1,2,3,4}$ and $R_{65}$ to describe the general R-matrix for the static magnetic beamline.

Matrices of the form (3.32) not only preserve itself under the multiplication, but form a subgroup in the group of 6 by 6 symplectic matrices. It is useful to point out that if the dispersive vector $\mathbf{D}$ is zero so is $\mathbf{E}$.

For a periodic system, we need to find the periodic dispersions, $\eta_{x}, \eta_{p_{x}}, \eta_{y}, \eta_{p_{y}}$ by the condition of the fixed point:

$$
\left(\begin{array}{l}
\eta_{x} \\
\eta_{p_{x}} \\
\eta_{y} \\
\eta_{p_{y}} \\
1
\end{array}\right)=\left(\begin{array}{lllll}
R_{11} & R_{12} & R_{13} & R_{14} & D_{1} \\
R_{21} & R_{22} & R_{23} & R_{24} & D_{2} \\
R_{31} & R_{32} & R_{33} & R_{34} & D_{3} \\
R_{41} & R_{42} & R_{43} & R_{44} & D_{4} \\
0 & 0 & 0 & 0 & 1
\end{array}\right) \cdot\left(\begin{array}{l}
\eta_{x} \\
\eta_{p_{x}} \\
\eta_{y} \\
\eta_{p_{y}} \\
1
\end{array}\right)
$$

It is obvious that the solution is given by,

$$
\boldsymbol{\eta}=(\mathbf{I}-\mathbf{T})^{-1} \cdot \mathbf{D}
$$

where $\eta$ is the vector of the periodical dispersion,

$$
\boldsymbol{\eta}=\left(\begin{array}{l}
\eta_{x} \\
\eta_{p_{x}} \\
\eta_{y} \\
\eta_{p_{y}}
\end{array}\right)
$$


and $\mathbf{I}$ is the $4 \times 4$ identity matrix. Knowing the periodic dispersions, we can make a similarity transformation,

$$
A_{\eta}^{-1} \cdot R \cdot A_{\eta}=R_{\eta}=\left(\begin{array}{llllll}
R_{11} & R_{12} & R_{13} & R_{14} & 0 & 0 \\
R_{21} & R_{22} & R_{23} & R_{24} & 0 & 0 \\
R_{31} & R_{32} & R_{33} & R_{34} & 0 & 0 \\
R_{41} & R_{42} & R_{43} & R_{44} & 0 & 0 \\
0 & 0 & 0 & 0 & 1 & 0 \\
0 & 0 & 0 & 0 & \lambda & 1
\end{array}\right),
$$

to take out the dispersion in the R-matrix. $R_{\eta}$ retains symplecticity because we have used a symplectic matrix,

$$
A_{\eta}=\left(\begin{array}{llllll}
1 & 0 & 0 & 0 & \eta_{x} & 0 \\
0 & 1 & 0 & 0 & \eta_{p_{x}} & 0 \\
0 & 0 & 1 & 0 & \eta_{y} & 0 \\
0 & 0 & 0 & 1 & \eta_{p_{y}} & 0 \\
0 & 0 & 0 & 0 & 1 & 0 \\
\eta_{p_{x}} & -\eta_{x} & \eta_{p_{y}} & -\eta_{y} & 0 & 1
\end{array}\right)
$$

for the transformation. Given the block form of $R_{\eta}$, it is easy to see by applying Eq. (3.25), we have

$$
R_{\eta}=A_{w} \cdot A_{\beta} \cdot F \cdot A_{\beta}^{-1} \cdot A_{w}^{-1},
$$

where

$$
\begin{aligned}
A_{w} & =\left(\begin{array}{cccccc}
g & 0 & w_{4} & -w_{2} & 0 & 0 \\
0 & g & -w_{3} & w_{1} & 0 & 0 \\
-w_{1} & -w_{2} & g & 0 & 0 & 0 \\
-w_{3} & -w_{4} & 0 & g & 0 & 0 \\
0 & 0 & 0 & 0 & 1 & 0 \\
0 & 0 & 0 & 0 & 0 & 1
\end{array}\right), \\
A_{\beta} & =\left(\begin{array}{ccccccc}
\sqrt{\beta_{1}} & 0 & 0 & 0 & 0 & 0 \\
\frac{-\alpha_{1}}{\sqrt{\beta_{1}}} & \frac{1}{\sqrt{\beta_{1}}} & 0 & 0 & 0 & 0 \\
0 & 0 & \sqrt{\beta_{2}} & 0 & 0 & 0 \\
0 & 0 & \frac{-\alpha_{2}}{\sqrt{\beta_{2}}} & \frac{1}{\sqrt{\beta_{2}}} & 0 & 0 \\
0 & 0 & 0 & 0 & 1 & 0 \\
0 & 0 & 0 & 0 & 0 & 1
\end{array}\right)
\end{aligned}
$$


and

$$
F\left(\mu_{1}, \mu_{2}, \lambda\right)=\left(\begin{array}{cccccc}
\cos \mu_{1} & \sin \mu_{1} & 0 & 0 & 0 & 0 \\
-\sin \mu_{1} & \cos \mu_{1} & 0 & 0 & 0 & 0 \\
0 & 0 & \cos \mu_{2} & \sin \mu_{2} & 0 & 0 \\
0 & 0 & -\sin \mu_{2} & \cos \mu_{2} & 0 & 0 \\
0 & 0 & 0 & 0 & 1 & 0 \\
0 & 0 & 0 & 0 & \lambda & 1
\end{array}\right)
$$

Here $F$ is the normal form of the periodic system and more importantly is invariant. Furthermore, it has the property of an abelian group,

$$
F\left(\phi_{1}, \psi_{1}, \lambda_{1}\right) \cdot F\left(\phi_{2}, \psi_{2}, \lambda_{2}\right)=F\left(\phi_{1}+\phi_{2}, \psi_{1}+\psi_{2}, \lambda_{1}+\lambda_{2}\right) .
$$

Combining Eqs. (3.40) and (3.42), we have

$$
R=A_{0} \cdot F \cdot A_{0}^{-1},
$$

where

$$
A_{0}=A_{\eta} \cdot A_{w} \cdot A_{\beta}
$$

Due to the abelian property of $F, A_{0}$ is far from unique and a more general transformation matrix is given by,

$$
A=A_{\eta} \cdot A_{w} \cdot A_{\beta} \cdot F\left(\psi_{1}, \psi_{2}, v\right) .
$$

It is easy to see that the matrix $A$ contains fifteen parameters. They are the independent parameters we choose to describe the linear optics when the beamline is entirely constructed from static magnets. We should point out that this kind of factorization and parameterization was first proposed by Ohmi, Hirata, and Oide [23]. The main difference is our normal form in the third dimension is not a rotation since we consider only the optics in a static magnetic beamline.

Once we have the optics parameters at a position $s_{1}$, we can easily compute the parameters at another position $s_{2}$ by propagating,

$$
A\left(s_{2}\right)=R_{(1 \rightarrow 2)} \cdot A_{0}\left(s_{1}\right),
$$

where $R_{(1 \rightarrow 2)}$ is the R-matrix from $s_{1}$ to $s_{2}$. Given the matrix $A\left(s_{2}\right)$, we can obtain the dispersion vector at $s_{2}$ using Eq. (3.49). The result is,

$$
\boldsymbol{\eta}=\left(\begin{array}{c}
A_{15} \\
A_{25} \\
A_{35} \\
A_{45}
\end{array}\right)
$$


And $\beta_{1,2}, \alpha_{1,2}, \psi_{1,2}$, and $w_{1,2,3,4}$ can be extracted by applying the procedure we derived in the previous subsection. Finally, $v$ can be obtained by using Eq. (3.49) again once all other parameters are calculated.

\section{Chromatic optics}

It is well known that the formulation we have reviewed in the previous section is applicable to the off-momentum particle as well. In this case, the fifteen parameters, $\beta_{1,2}, \alpha_{1,2}, \psi_{1,2}, \eta_{x, p_{x}, y, p_{y}}, w_{1,2,3,4}$ and $v$ are all functions of $\delta$. In fact, we know how to compute them numerically based on the linear optics. In this section, we would like to develop an analytical method to calculate these functions. Since this is a nonlinear problem, an obvious point is to start with the transfer map of the beamline,

$$
\mathcal{M}=\mathcal{M}_{1} \circ \mathcal{M}_{2} \ldots \circ \mathcal{M}_{n}
$$

where $\mathcal{M}_{i}$ is the transfer map of the $i^{\text {th }}$ element and index 1 is for the first element seen by the charged particle and $n$ for the last. Here we have defined the concatenation of the map by,

$$
\mathcal{M}_{1} \circ \mathcal{M}_{2}\left(z\left(s_{1}\right)\right) \equiv \mathcal{M}_{2}\left(\mathcal{M}_{1}\left(z\left(s_{1}\right)\right)\right)
$$

where $z$ is the vector of phase space variables we have introduced in Sect. 2 . Essentially, the concatenation of two maps can be computed by substituting the first map into the second one. It is easy to show that the concatenation preserves the symplecticity. This particular order of maps in the beamline is chosen so that it is consistent with the order of Lie operators. Moreover, this order actually is consistent with the multiplication of the R-matrix for the beamline in the previous section.

Given the map $\mathcal{M}$, we introduce an operator,

$$
\left.\mathcal{J}[\mathcal{M}] \equiv \mathcal{J}(\mathcal{M})\right|_{x=0, p_{x}=0, y=0, p_{y}=0, \ell=0} .
$$

Here the symbol $\mathcal{J}(\mathcal{M})$ represents taking the Jacobian of the map $\mathcal{M}$ as defined in Eq. (2.1). It is obvious that this operation turns the map to a symplectic matrix $R(\delta)$ with $\delta$ dependence. Moreover, $R(0)$ is the same as the R-matrix we have extensively used in the previous section. For this reason, we consider it as a natural extension of the R-matrix and use it as a starting point. 
For a periodic system, we need to find the periodic dispersion as a function of $\delta$ and the transfer map relative to the dispersive orbit. This can be achieved by an iterative loop of index i starting from 0 with $\mathcal{M}^{0}=\mathcal{M}$. For each index i, we compute,

$$
R(\delta)=\mathcal{J}\left[\mathcal{M}^{i}\right] .
$$

Expanding the matrix $R(\delta)$ as a power series of $\delta$, we find that the lowest non-vanishing dispersive elements are given by,

$$
D^{(i)}=\left(\begin{array}{c}
R_{15}^{(i)} \\
R_{25}^{(i)} \\
R_{35}^{(i)} \\
R_{45}^{(i)}
\end{array}\right),
$$

where the superscript $(i)$ represents the coefficient of the $i^{\text {th }}$ power of $\delta$. Similar to the linear case in the previous section, the periodic dispersion at this order of $\delta$ is given by,

$$
\boldsymbol{\eta}^{(i)}=(\mathbf{I}-\mathbf{T})^{-1} \cdot \mathbf{D}^{(i)},
$$

where the matrices $\mathbf{I}$ and $\mathbf{T}$ are the same as those in Eq. (3.38). To remove the ith-order dispersion, we perform,

$$
\mathcal{M}^{i+1}=\mathcal{A}_{\eta^{i}} \circ \mathcal{M}^{i} \circ \mathcal{A}_{\eta^{i}}^{-1},
$$

where we have chosen the map $\mathcal{A}_{\eta^{i}}$,

$$
\begin{aligned}
& \mathcal{A}_{\eta^{i} 1}=x+\eta_{x}^{(i)} \frac{\delta^{i+1}}{(i+1)}, \\
& \mathcal{A}_{\eta^{i} 2}=p_{x}+\eta_{p_{x}}^{(i)} \frac{\delta^{i+1}}{(i+1)}, \\
& \mathcal{A}_{\eta^{i} 3}=y+\eta_{y}^{(i)} \frac{\delta^{i+1}}{(i+1)}, \\
& \mathcal{A}_{\eta^{i} 4}=p_{y}+\eta_{p_{y}}^{(i)} \frac{\delta^{i+1}}{(i+1)}, \\
& \mathcal{A}_{\eta^{i} 5}=\delta \\
& \mathcal{A}_{\eta^{i} 6}=\ell+\left(\eta_{p_{x}}^{(i)} x-\eta_{x}^{(i)} p_{x}+\eta_{p_{y}}^{(i)} y-\eta_{y}^{(i)} p_{y}\right) \delta^{i},
\end{aligned}
$$


so that $\mathcal{J}\left[\mathcal{A}_{\eta^{i}}\right]$ is consistent with $A_{\eta}$ in Eq. (3.41). Its inverse, $\mathcal{A}_{\eta^{i}}^{-1}$ can be obtained by simply changing the sign of $\boldsymbol{\eta}^{(i)}(\delta)$ in Eq. (4.8). It is easy to see that this map is symplectic. As a result, the map $\mathcal{M}^{i+1}$ retains its symplecticity under the similarity transformation. It is worth noting that this transformation leads to the chromaticity generated by sextupoles through the dispersions. Here we repeat the process with the next index: $i+1$.

It is easy to check that the first step leads to identical results to those we have shown in the previous section in the matrix formulation. At the end of the loop, we have the periodic dispersion vector,

$$
\boldsymbol{\eta}(\delta)=\sum_{i=0}^{\infty} \boldsymbol{\eta}^{(i)} \delta^{i}
$$

and the map,

$$
\mathcal{M}_{\eta}=\mathcal{A}_{\eta} \circ \mathcal{M} \circ \mathcal{A}_{\eta}^{-1}
$$

where the total dispersive map is given by,

$$
\mathcal{A}_{\eta}=\mathcal{A}_{\eta^{\infty}} \ldots \mathcal{A}_{\eta^{1}} \circ \mathcal{A}_{\eta^{0}}
$$

Here $\mathcal{M}_{\boldsymbol{\eta}}$ is the transfer map relative to the dispersive orbit described by $\mathcal{A}_{\eta}$. This transfer map contains the physics of beam dynamics relative to the closed orbit, including the chromatic optics and the effects of the betatron resonances. Applying the Jacobian operator on the map,

$$
R_{\eta}(\delta)=\mathcal{J}\left[\mathcal{M}_{\boldsymbol{\eta}}\right]
$$

we extract the pure chromatic physics near the vicinity of the orbit but lose the information of the geometric and geometric-chromatic resonances. Since the resonances are not a subject in this paper, the loss is acceptable. It is clear from our construction that $R_{\eta}(0)$ equals $R_{\eta}$ in Eq. (3.40). It can be shown that all formulas, used in the linear optics for computing the parameters: $\beta_{1,2}, \alpha_{1,2}, \mu_{1,2}, w_{1,2,3,4}$, and $\lambda$, retain their functional forms. However now, they are all a function of $\delta$.

In order to propagate the chromatic optical parameters, we compute a matrix $A_{0}(\delta)$ at position $s_{1}$ by applying Eq. (3.48) with $\delta$ dependence and then construct a map $\mathcal{A}_{0}$ so that $\mathcal{J}\left(\mathcal{A}_{0}\right)=A_{0}(\delta)$. Now the map $\mathcal{A}\left(s_{2}\right)$ can be obtained by,

$$
\mathcal{A}\left(s_{2}\right)=\mathcal{A}_{0}\left(s_{1}\right) \circ \mathcal{M}_{(1 \rightarrow 2)},
$$


where $\mathcal{M}_{(1 \rightarrow 2)}$ is the transfer map from the position $s_{1}$ to $s_{2}$. Finally, the matrix $A(\delta)$ at $s_{2}$ is given by,

$$
A(\delta)=\mathcal{J}\left[\mathcal{A}\left(s_{2}\right)\right],
$$

which can be factorized as in Eq. (3.49). The factorization allows us to compute all the lattice parameters at position $s_{2}$.

Again, this simple procedure allows us to propagate the lattice functions through a beamline. For a periodic system, the initial condition is determined by the periodic condition. The process of finding the initial condition is essentially a procedure to derive the transformation to the normal form. In a single-pass beamline, its initial condition can be constructed as some initial parameters. As a result, the chromatic lattice parameters down to the beamline are functions of the initial inputs.

\section{Application}

In this section, we would like to illustrate how the scheme works using a periodic alternating-gradient cell as an example. The cell is chosen because it contains the most essential ingredients in storage rings. For simplicity, we will use the maps given in Sec. 2 .

\subsection{Cell with sextupoles}

A schematic drawing of the alternating focusing and de-focusing (FODO) cell is shown in Fig. 2. The quadrupoles and sextupoles are lumped together as a thin multipole with a sector bending dipole in between. Here $f$ is the focal length of quadrupoles. Also $\phi$ is the total bending angle and $L$ the total length of the cell.

The cell starts at the center of the first focusing (in the horizontal plane) quadrupole with $s=0$ and ends at the middle of the next focusing quadrupole with $s=L$. The transfer map of the cell can be computed through the concatenation of the maps of the elements. Here, we use Mathematica [24] to perform the calculation. With the map, we start at the first step of the iteration loop as we outlined in the previous section and find that the betatrons phase advances in both planes are the same $\mu_{x}=\mu_{y}=\mu$ and given by,

$$
\sin \frac{\mu}{2}=\frac{L}{4 f}
$$




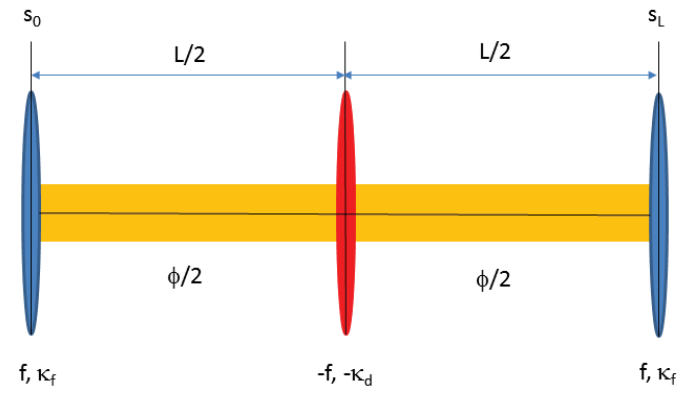

Figure 2: A periodic focusing and de-focusing cell with dipole, quadrupole, and sextuploe magnets.

and the beta functions at $s=0$,

$$
\begin{aligned}
& \beta_{x}=\frac{L\left(1+\sin \frac{\mu}{2}\right)}{\sin \mu}, \\
& \beta_{y}=\frac{L\left(1-\sin \frac{\mu}{2}\right)}{\sin \mu},
\end{aligned}
$$

and the horizontal dispersion,

$$
\eta_{x}=\frac{L \phi\left(1+\frac{1}{2} \sin \frac{\mu}{2}\right)}{4 \sin ^{2} \frac{\mu}{2}} .
$$

$\alpha_{x, y}=0$ and $\eta_{p_{x}}=0$ due to the reflectional symmetry and $\eta_{y, p_{y}}=0$ since the bending is in the horizontal plane and there are no coupling elements. Our results here are the same as those in the standard reference [25].

With the zeroth-order dispersion, we proceed to the next iteration by performing the similarity transformation to the transfer map. Following the procedure, we find that the phase advances up to the first-order expansion 
of $\delta$ are

$$
\begin{aligned}
\mu_{x}(\delta)= & \mu-\tan \frac{\mu}{2}\left[2-\frac{1}{4 \sin \frac{\mu}{2}}\left(\frac{1}{2}+\frac{1}{\sin ^{2} \frac{\mu}{2}}\right)\left(\kappa_{f}-\kappa_{d}\right) f L \phi\right. \\
& \left.-\frac{3}{8 \sin ^{2} \frac{\mu}{2}}\left(\kappa_{f}+\kappa_{d}\right) f L \phi\right] \delta, \\
\mu_{y}(\delta)= & \mu-\tan \frac{\mu}{2}\left[2-\frac{1}{4 \sin \frac{\mu}{2}}\left(\frac{1}{2}-\frac{1}{\sin ^{2} \frac{\mu}{2}}\right)\left(\kappa_{f}-\kappa_{d}\right) f L \phi\right. \\
& \left.-\frac{1}{8 \sin ^{2} \frac{\mu}{2}}\left(\kappa_{f}+\kappa_{d}\right) f L \phi\right] \delta,
\end{aligned}
$$

where $\kappa_{f, d}$ are the integrated strengths of the sextupoles. The linear coefficient of $\delta$ dividing by $2 \pi$ gives us the chromaticity. Clearly, the natural chromaticities in both planes are the same and given by, $\xi_{0}=-\frac{1}{\pi} \tan \frac{\mu}{2}$, which agrees with that in the handbook [25]. Obviously, we can use the two sextupoles to cancel the natural chromaticities. Solving two linear equations, we find the sextupole strengths,

$$
\begin{aligned}
\kappa_{f} & =\frac{4 \sin ^{2} \frac{\mu}{2}}{f L \phi\left(1+\frac{1}{2} \sin \frac{\mu}{2}\right)}, \\
\kappa_{d}= & \frac{4 \sin ^{2} \frac{\mu}{2}}{f L \phi\left(1-\frac{1}{2} \sin \frac{\mu}{2}\right)} .
\end{aligned}
$$

Rewriting them in terms of the dispersions, we have that $\kappa_{f}=1 /\left(\eta_{x} f\right), \kappa_{d}=$ $1 /\left(\eta_{x}^{d} f\right)$ where $\eta_{x}^{d}$ is the dispersion at the position of the defocusing quadrupole. Intuitively, we know that they are the correct settings for the local chromatic compensation. It is obvious that the sextupole at the de-focusing quadrupole is stronger than the one at the focusing quadrupole.

Now we set the sextupole to the values in Eq. (5.5) and continue to find the first-order dispersion,

$$
\begin{aligned}
\eta_{x}^{(1)}(\delta) & =-\frac{f \phi}{2} \delta \\
\eta_{p_{x}}^{(1)}(\delta) & =0
\end{aligned}
$$

with which we proceed to the next iteration by performing another similarity transformation to the map that we obtained in the previous step. Continuing with the procedure, we find that the phase advances up to the second-order 
expansion of $\delta$ are

$$
\begin{aligned}
& \mu_{x}(\delta)=\mu-\frac{\tan \frac{\mu}{2}\left(1-\frac{1}{2} \sin ^{2} \frac{\mu}{2}\right)}{2\left(1-\frac{1}{4} \sin ^{2} \frac{\mu}{2}\right)} \delta^{2} \\
& \mu_{y}(\delta)=\mu+\frac{\tan \frac{\mu}{2}\left(1+\frac{1}{2} \sin ^{2} \frac{\mu}{2}\right)}{2\left(1-\frac{1}{4} \sin ^{2} \frac{\mu}{2}\right)} \delta^{2} .
\end{aligned}
$$

Although the linear parts have been corrected by the sextupoles, there are the second-order chromatic effects. Correspondingly, we have the beta functions,

$$
\begin{aligned}
& \beta_{x}(\delta)=\beta_{x}\left[1-\delta+\frac{10-13 \sin ^{2} \frac{\mu}{2}-\sin ^{3} \frac{\mu}{2}+3 \sin ^{4} \frac{\mu}{2}}{2\left(4-5 \sin ^{2} \frac{\mu}{2}+\sin ^{4} \frac{\mu}{2}\right)} \delta^{2}\right] \\
& \beta_{y}(\delta)=\beta_{y}\left[1-\delta+\frac{3\left(2-3 \sin ^{2} \frac{\mu}{2}-\sin ^{3} \frac{\mu}{2}+\sin ^{4} \frac{\mu}{2}\right)}{2\left(4-5 \sin ^{2} \frac{\mu}{2}+\sin ^{4} \frac{\mu}{2}\right)} \delta^{2}\right] .
\end{aligned}
$$

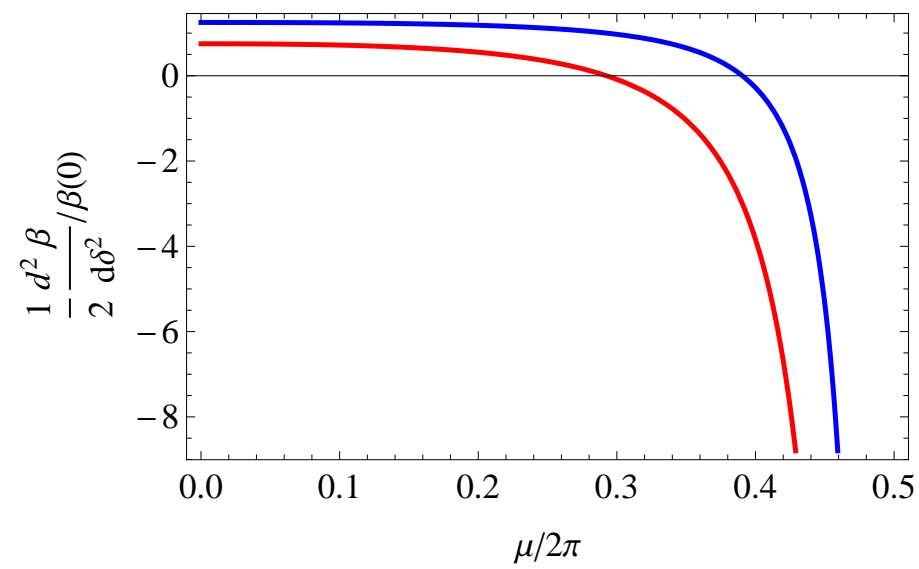

Figure 3: The second-derivative of chromatic beta relative to the ideal beta as a function of the betatron tune in FODO cell. The horizontal values are plotted in blue color and the vertical in red.

Using these formulas, we show the second-order chromatic effects in Fig. 3. From the plots, we see clearly that the beta beatings are rather small when the phase advances are less than 90 degrees. However, their amplitudes steeply increase beyond 135 degrees. This sharp increase of chromatic beta 
function is called the chromaticity wall, which may well be the reason why the high-tune FODO cells were never used in circular accelerators. Theoretically, the cause of the wall is due to the half integer resonance, at which the denominator of the second-order coefficients is zero.

Moreover, the horizontal dispersion functions are given by,

$$
\eta_{x}(\delta)=\eta_{x}\left[1-\frac{\sin \frac{\mu}{2}}{2\left(1+\frac{1}{2} \sin \frac{\mu}{2}\right)}\left(\delta-\frac{3}{2} \delta^{2}\right)\right],
$$

and finally, the invariance in the longitudinal plane,

$$
\lambda(\delta)=L\left(\frac{\phi}{2}\right)^{2}\left[\left(\frac{1}{\sin ^{2} \frac{\mu}{2}}-\frac{1}{12}\right)+\left(\frac{1}{\sin ^{2} \frac{\mu}{2}}+\frac{1}{4}\right) \delta-\frac{3}{2}\left(\frac{1}{\sin ^{2} \frac{\mu}{2}}+\frac{1}{3}\right) \delta^{2}\right] .
$$

Here for a ultra-relativistic particle, we have the momentum compaction factor $\alpha_{p}(\delta)=\lambda(\delta) / L$. Its zeroth-order component agrees with that in the handbook [25].

In order to compute the lattice functions at the other positions in the FODO cell, we start with the map $\mathcal{A}_{0}$,

$$
\begin{aligned}
& \mathcal{A}_{01}=\sqrt{\beta_{x}(\delta)} x+\int_{0}^{\delta} \eta_{x}\left(\delta^{\prime}\right) d \delta^{\prime} \\
& \mathcal{A}_{02}=\frac{1}{\sqrt{\beta_{x}(\delta)}} p_{x} \\
& \mathcal{A}_{03}=\sqrt{\beta_{y}(\delta)} y \\
& \mathcal{A}_{04}=\frac{1}{\sqrt{\beta_{y}(\delta)}} p_{y} \\
& \mathcal{A}_{05}=\delta, \\
& \mathcal{A}_{06}=\ell-\frac{\eta_{x}(\delta)}{\sqrt{\beta_{x}(\delta)}} p_{x} .
\end{aligned}
$$

The initial map is so simple because of the symmetry. Substituting it into the transfer map $\mathcal{M}_{0 \rightarrow s}$, we find the beta functions at the position $s$,

$$
\begin{aligned}
& \beta_{x}(\delta, s)=\beta_{x}\left\{\left[1-\frac{4 s}{L} \sin \frac{\mu}{2}\left(1-\frac{2 s \sin \frac{\mu}{2}}{L\left(1+\sin \frac{\mu}{2}\right)}\right)\right](1-\delta)\right\} \ldots, \\
& \beta_{y}(\delta, s)=\beta_{y}\left\{\left[1+\frac{4 s}{L} \sin \frac{\mu}{2}\left(1+\frac{2 s \sin \frac{\mu}{2}}{L\left(1-\sin \frac{\mu}{2}\right)}\right)\right](1-\delta)\right\} \ldots,
\end{aligned}
$$


where $s$ is the distance away from the center of the focusing quadrupole. We have omitted the second-order terms because they are too lengthy to fit into the page. These formulas are only valid between the focusing and de-focusing quadrupoles where $0<s<L / 2$. The expressions in the other half of the cell can be obtained by substituting $s$ with $L-s$ in Eq. (5.12).
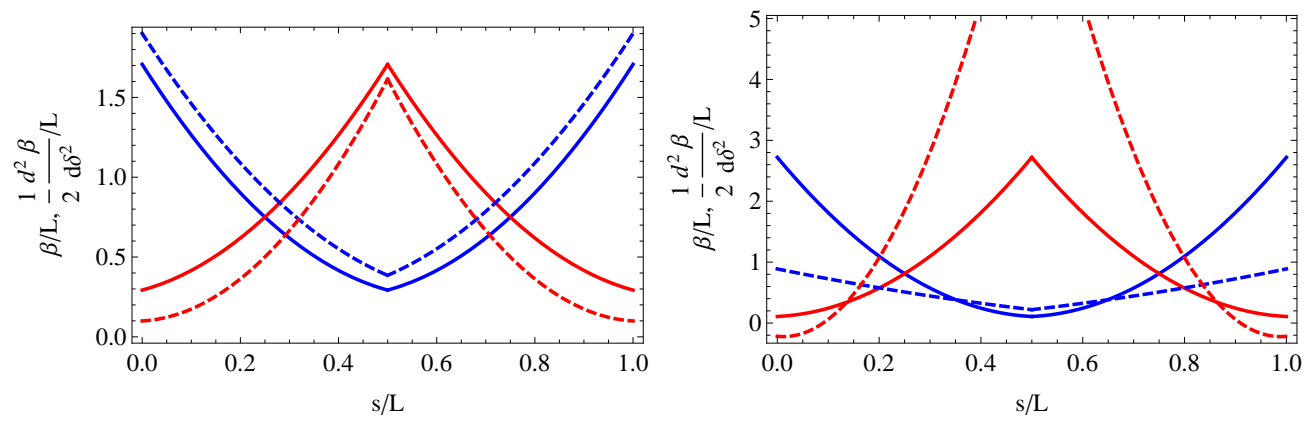

Figure 4: The beta function and its second derivative as a function of the position in the FODO cells with $90^{\circ}$ (on left) and $135^{\circ}$ (on right) phase advances. The horizontal values are plotted in blue color and the vertical in red. The solid lines represent the beta functions and dashed lines for their second derivatives.

The beta functions and their second derivative with respect to $\delta$ are shown in Fig. 4 for the FODO cells with $90^{\circ}$ and $135^{\circ}$ phase advances. The figure clearly demonstrates the alternating focusing principle [1] for the on-momentum particle. However for the off-momentum particles, there are chromatic beatings that grow larger as the focusing becomes stronger. Based on these results, we conclude that the chromatic optics in the $90^{\circ}$ cell is well behaved. This may explain why it is one of the mostly widely used cells in the circular colliders such as LHC.

As one can see from the analysis, the second-order chromatic effects in the FODO cell becomes asymmetric between the horizontal and vertical planes. The vertical plane is worse presumably because of the stronger sextupole at the de-focusing quadupole.

To check the analytical results against numerical computation, we construct a ring that consists of $N_{c}=101$ cells with $90^{\circ}$ phase advance per cell and zero chromaticities. Here we use the cell length $L=15.0$ meter and bending angle $\phi=2 \pi / N_{c}$. The betatron tunes of the ring are computed as a function of the momentum deviation $\delta$ using LEGO [26]. The results 
are plotted in Fig. 5 against the analytical formula $\nu_{x, y}(\delta)=N_{c} \mu_{x, y}(\delta) /(2 \pi)$, where the cell phase advances $\mu_{x, y}(\delta)$ are given in Eq. (5.7). It is clear from the figure that the agreement is excellent. Essentially, the second-order expansion in $\delta$ gives us a good description of the chromatic optics for the FODO cells.
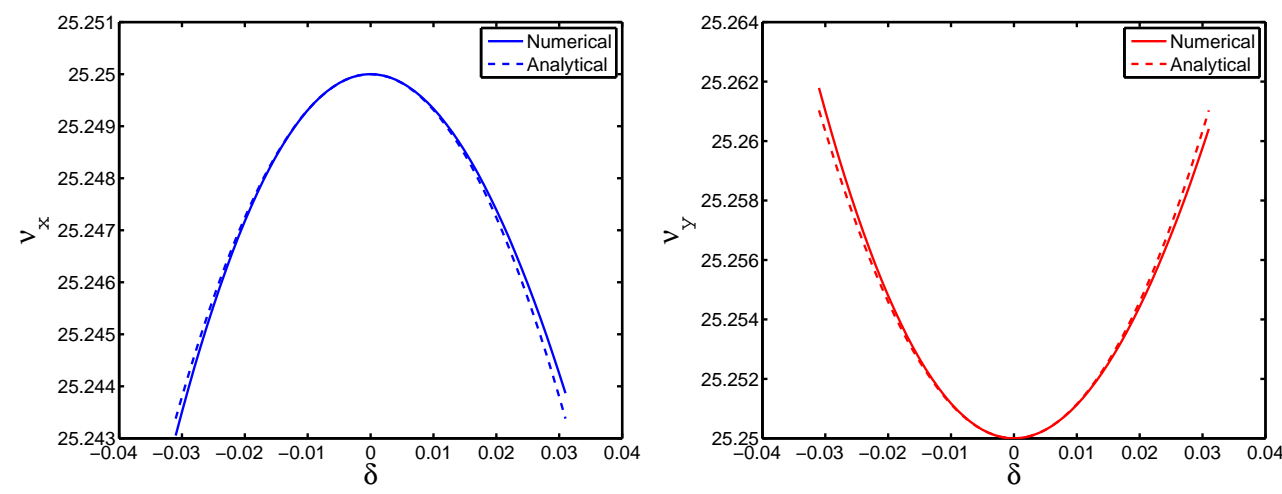

Figure 5: Betatron tunes as a function of the momentum deviation $\delta$ in a storage ring that consists of $90^{\circ}$ FODO cells.

\subsection{Octupoles and decapoles}

Note that the changes in tunes are so small in Fig. (5). It shows that the traditional chromatic compensation using a pair of sextupoles in a FODO cell is quite adequate. Nevertheless, we would like to show how to further improve the chromatic optics by introducing a pair of octupoles and decapoles at the locations of quadupoles and sextupoles in the FODO cell. Continuing on to carry out the next two steps in the perturbation of $\delta$, we find that the second-order coefficients of the phase advances in Eq. (5.7) can be eliminated by setting the octupole strengths:

$$
\begin{aligned}
o_{f} & =\frac{8 \sin ^{5} \frac{\mu}{2}}{f L^{2} \phi^{2}\left(1+\frac{1}{2} \sin \frac{\mu}{2}\right)^{3}}, \\
o_{d} & =-\frac{8 \sin ^{5} \frac{\mu}{2}}{f L^{2} \phi^{2}\left(1-\frac{1}{2} \sin \frac{\mu}{2}\right)^{3}},
\end{aligned}
$$


and then the third-order by the pair of decapoles with their strengths:

$$
\begin{aligned}
& \xi_{f}=\frac{16 \sin ^{6} \frac{\mu}{2}\left(4-2 \sin \frac{\mu}{2}+\sin ^{2} \frac{\mu}{2}\right)}{f L^{3} \phi^{3}\left(1+\frac{1}{2} \sin \frac{\mu}{2}\right)^{5}}, \\
& \xi_{d}=\frac{16 \sin ^{6} \frac{\mu}{2}\left(4+2 \sin \frac{\mu}{2}+\sin ^{2} \frac{\mu}{2}\right)}{f L^{3} \phi^{3}\left(1-\frac{1}{2} \sin \frac{\mu}{2}\right)^{5}} .
\end{aligned}
$$

Due to the feed-down effects from the high-order dispersions, the interpretations of the these settings are not as simple as the sextupoles'. In general, the feed-down makes their strengths weaker as shown in Fig. 6.
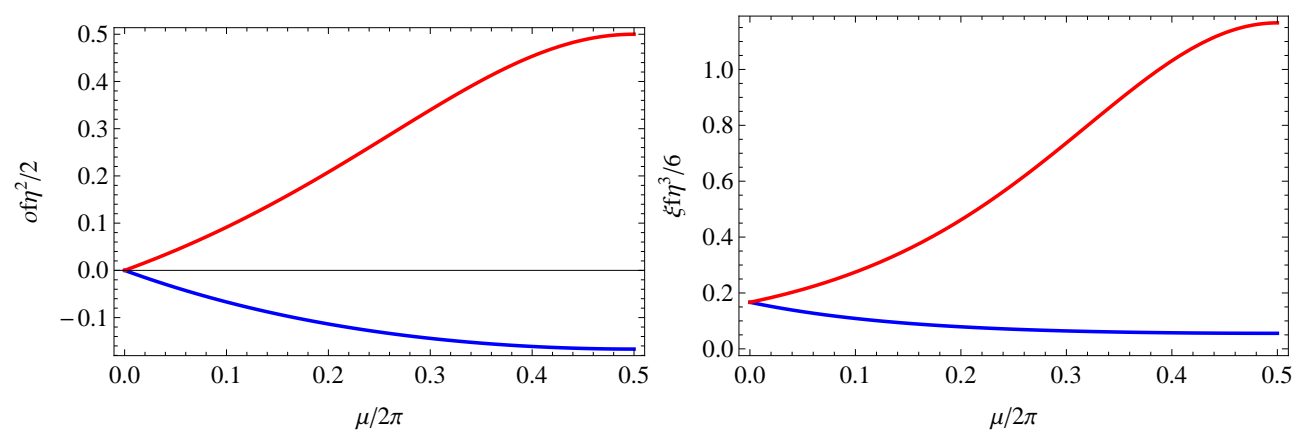

Figure 6: Strengths of octupoles (on left) and decapoles (on right) as a function of the tune in FODO cells. The horizontal values are plotted in blue color and the vertical in red.

As a result of the octupole and decapole correctors, the phase advances up to the fourth-order expansion of $\delta$ become

$$
\begin{aligned}
& \mu_{x}(\delta)=\mu+\frac{\tan \frac{\mu}{2}\left(-352+312 \sin ^{2} \frac{\mu}{2}+60 \sin ^{4} \frac{\mu}{2}+\sin ^{6} \frac{\mu}{2}\right) \delta^{4}}{12\left(4-\sin ^{2} \frac{\mu}{2}\right)^{3}} \\
& \mu_{y}(\delta)=\mu+\frac{\tan \frac{\mu}{2}\left(992+840 \sin ^{2} \frac{\mu}{2}+84 \sin ^{4} \frac{\mu}{2}+\sin ^{6} \frac{\mu}{2}\right) \delta^{4}}{12\left(4-\sin ^{2} \frac{\mu}{2}\right)^{3}} .
\end{aligned}
$$

These analytical formulae of the tunes are compared in Fig. 7 against the numerical computation of those in the FOOD ring we have constructed previously with the settings of octupoles and decapoles respectively in Eqs. (5.13) and (5.14). Again the agreement is excellent and the tune curves are flattened up to the fourth-order of $\delta$. 

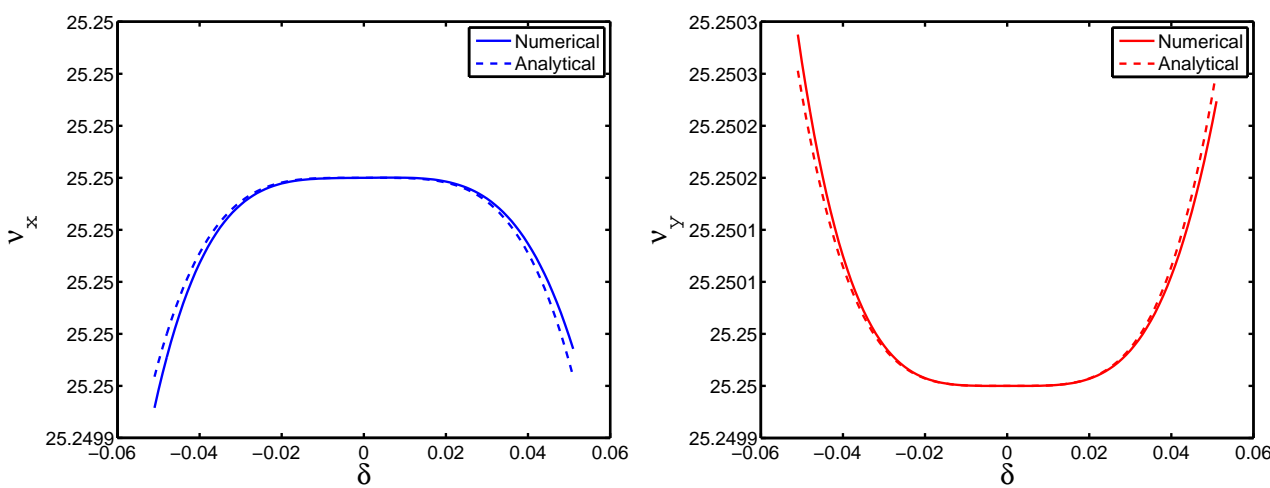

Figure 7: Betatron tunes as a function of the momentum deviation $\delta$ in a storage ring that consists of $90^{\circ}$ FODO cells with the high-order chromatic correction.

Moreover, the beta functions in the region, $0<s<L / 2$, are given by

$$
\begin{aligned}
& \beta_{x}(\delta, s)=\beta_{x}\left[1-\frac{4 s}{L} \sin \frac{\mu}{2}\left(1-\frac{2 s \sin \frac{\mu}{2}}{L\left(1+\sin \frac{\mu}{2}\right)}\right)\right]\left(1-\delta+\delta^{2}-\delta^{3}\right), \\
& \beta_{y}(\delta, s)=\beta_{y}\left[1+\frac{4 s}{L} \sin \frac{\mu}{2}\left(1+\frac{2 s \sin \frac{\mu}{2}}{L\left(1-\sin \frac{\mu}{2}\right)}\right)\right]\left(1-\delta+\delta^{2}-\delta^{3}\right) .
\end{aligned}
$$

Here again we do not write the fourth-order terms because of their long length. Correspondingly, the phase advances up to the third-order can be written as,

$$
\begin{aligned}
& \psi_{x}(\delta, s)=\tan ^{-1}\left[\frac{\tan \frac{\mu}{2}\left(1-\sin \frac{\mu}{2}\right) \frac{2 s}{L}}{1-\frac{2 s}{L} \sin \frac{\mu}{2}}\right] \\
& \psi_{y}(\delta, s)=\tan ^{-1}\left[\frac{\tan \frac{\mu}{2}\left(1+\sin \frac{\mu}{2}\right) \frac{2 s}{L}}{1+\frac{2 s}{L} \sin \frac{\mu}{2}}\right] .
\end{aligned}
$$

Amazingly, there is no dependence on $\delta$. We should mention that the highorder terms in dispersion at $s=0$ are perturbed a little by the octupoles and decaples and now is given by,

$$
\eta_{x}(\delta)=\frac{L \phi}{8 \sin ^{2} \frac{\mu}{2}}\left[\left(2+\sin \frac{\mu}{2}\right)-\sin \frac{\mu}{2} \delta+\left(1-\sin \frac{\mu}{2}\right) \delta^{2}+\frac{1}{3}\left(5-3 \sin \frac{\mu}{2}\right) \delta^{3}\right] .
$$

The chromatic correction can be carried out to the next order. Since no one has yet used any multipoles beyond the decapole, we choose to stop here. 
As we have shown in this section, it is better to treat the chromatic optics as a perturbation theory in the expansion of $\delta$. Moreover, this approach allows us to chromatically match to the other optical modules order-by-order. Naturally, this kind of expansion can be approximated by the differential algebra [12]. In fact, the order-by-order calculation of the chromatic optics outlined in this paper is implemented recently in LEGO [26] using the differential algebra technique.

Though the results of this section are given as Taylor expansions up to finite order in relative momentum deviation, the usage of Mathematica (in

contrast to the usage of the differential algebra) allows to have coefficients of these expansions in symbolic form.

\section{Conclusion}

We need a total of fifteen lattice parameters with dependence on the momentum deviation $\delta$ to characterize the chromatic optics in a beamline that consists of only the magnetic elements. These parameters can be propagated from one element to another through the symplectic transfer map. For a periodic system, their initial values are completely determined by the periodic condition. We have developed an iterative procedure with respect to the power expansion of $\delta$ to compute their values. In a single-pass system, the initial values can be assigned as input parameters. As a result, the lattice parameters in the beamline will have functional dependence on these input parameters. Aside from the symplectic transfer maps, the key ingredient is the Jacobian operator that links a map to a matrix with the dependence of $\delta$. And this matrix allows us to efficiently utilize the matrix theory of linear optics.

In a FODO cell with sextupoles, our analysis yields several insights to its chromatic optics. Moreover, we show that the higher-order chromaticities can be compensated by octupoles and decapoles. The nonlinear correction makes the cell nearly perfect up to the third-order of $\delta$.

A similar analysis can performed for the other type of cells such as the double-bend achromat. Hopefully, this kind of analytical approach will provide us more understanding of beam physics and lead to better designs of the next generation accelerators. 


\section{Acknowledgments}

I would like to thank Johan Bengtsson, Alex Chao, Etienne Forest, John Irwin, and Yiton Yan for many stimulating and helpful discusses. This work was supported by the Department of Energy under Contract Number: DEAC02-76SF00515.

\section{References}

[1] E. D. Courant and H. S. Snyder, "Theory of the Alternating-Gradient Synchrotron," Annals of Physics: 3, 1-48 (1958).

[2] D.A. Edwards and L.C. Teng, Trans. Nucl. Sci. 20, 885 (1973).

[3] K.L. Brown, "A First- and Second-Order Matrix Theory for the Design of Beam Transport Systems and Charged Particle Spectrometers," SLAC Rep. No 75; Adv. Particle Phys. 1 71-134 (1967).

[4] H. Grote and F. C. Iselin, "The MAD Program (Methodical Accelerator Design) Version 8.15," CERN/SL/90-13 (AP), (1990).

[5] M. Borland el at., "Multi-objective direct optimization of dynamic acceptance and lifetime for potential upgrades of the advanced photon light source," APS LS 319, Argonne, 2010.

[6] M. Donald and D. Schofield, "A User's Guild to the HARMON Program," LEP Note 420, CERN, 1982.

[7] K. Oide, "Strategic Accelerator Design," see SAD/Tkinker, KEK http://acc-physics.kek.jp/SAD/sad.html (1997).

[8] D.A. Edwards and M.J. Syphers, An introduction to the physics of high energy accelerators, John Wiley \& Sons, Inc. (1993).

[9] J. Bengtsson, "The Sextupole Scheme for the Swiss Light Source (SLS): An Analytic Approach," SLA Internal Report No. 9/97, March (1997).

[10] M. Berz, "Differential Algebra Description of Beam Dynamics to Very High Order," Part. Accel. 24, 109 (1989). 
[11] E. Forest, M. Berz, and J. Irwin, "Normal Form Methods for Complicated Periodic Systems: A Complete Solution Using Differential Algebra and Lie Operators," Part. Accel. 2491 (1989).

[12] M. Berz, Modern Map Methods in Particle Beam Physics, Academic Press, (1999).

[13] E. Forest, Beam Dynamics: A New Attitude and Framework, Hardwood Academic Publishers, (1998).

[14] A. Blondel et al., "LEP3 : A High Luminosity $e^{+} e^{-}$Collider to Study the Higgs Boson," CERN-ATS-NOTE-2012-061 TECH, August, 2012.

[15] V.I. Telnov, "Restriction on the energy and luminosity of $e^{+} e^{-}$storage rings due to beamstrahlung," Phys. Rev. Lett. 110, 114801 (2013).

[16] Y. Cai el al., "Ultimate storage ring based on fourth-order geometric achromats," Phys. Rev. ST, Accel. Beams, 15, 054002 (2012).

[17] R.D. Ruth, "Single Particle Dynamics in Circular Accelerator," AIP Conference Proceedings No. 153, Vol.1 p166, M. Month and M. Dienes editors (1985).

[18] F. Willeke and G. Ripken, "Methods of Beam Optics," DESY 88-114, (1988).

[19] Yunhai Cai, "Single-Particle Dynamics in Electron Storage Rings with Extremely Low Emittance," Nucl. Instrum. Methods Phys. Res. Sect. A 645, 168 (2011).

[20] J. Bengtsson, E. Forest, and H. Nishimura, "Tracy User Manual," Unpublised (ALS Berkeley).

[21] Karl L. Brown and Roger V. Servranckx, "First- and Second-Order Charged Particle Optics," SLAC-PUB-3381, July (1984).

[22] D. Sagan and D. Rubin, "Linear Analysis of Coupled Lattices," Phys. Rev. ST, Accel. Beams, 2, 074001 (1999).

[23] K. Ohmi, K. Hirata, and K. Oide, "From the Beam-Envelope Matrix to Synchrotron-Radiation Integrals," Phys. Rev. E 49, 751 (1994). 
[24] Mathematica version 9, "A System for Doing Mathematics by Computer," Wolfram Reaserch. Inc. (2014).

[25] E. Keil, "Lattices for Collider Storage Rings," p78 in Handbook of Accelerator Physics and Engineering, Second Edition, Edited by A. Chao, K.H. Mess, M. Tigner, F. Zimmermann, World Scietific, (2013).

[26] Y. Cai, M. Donald, J. Irwin, Y. Yan, "LEGO: A Modular Accelerator Design Code," SLAC-PUB-7642, August (1997). 